RESEARCH

Turkish Journal of Geriatrics

DOI: $10.31086 /$ tjgeri.2021.217

2021; 24(2): 212-219

\title{
FACTORS AFFECTING MORTALITY IN GERIATRIC PATIENTS DIAGNOSED WITH COMMUNITY-ACQUIRED PNEUMONIA TREATED IN INTENSIVE CARE UNITS
}

- Hatice Eylül BOZKURT YILMAZ ${ }^{1}$

- Zühal Ekici ÜNSAL ${ }^{2}$

- Mehmet Ali HABEŞOĞLU2

- Sibel KARA ${ }^{2}$

- Nazan ŞEN ${ }^{2}$

CORRESPONDANCE

${ }^{1}$ Hatice Eylül BOZKURT YILMAZ

Seyhan State Hospital, Pulmonary Medicine Clinic, Adana, Turkey

\section{Phone: +905337748023}

e-mail: b_eylul@yahoo.com

Received: Dec 10, 2020

Accepted: Apr 07, 2021

${ }^{1}$ Seyhan State Hospital, Pulmonary Medicine Clinic, Adana, Turkey

2 Baskent University, Faculty of Medicine, Pulmonary Medicine Clinic, Adana, Turkey

\section{Abstract}

Introduction: The aim of this study was to determine the factors affecting mortality in elderly patients with community-acquired pneumonia who were receiving intensive care unit.

Materials and Methods: The study was retrospective, cross-sectional, and descriptive. The medical records of patients over 65 years of age who were admitted to the intensive care unit with a diagnosis of community-acquired pneumonia between January 1, 2013 and February 29, 2020 were reviewed. The factors associated with mortality in the patients who had died were examined.

Results: A total of 208 patients with a mean age of $75.11 \pm 5.59$ years, 78 of whom were women (37.5\%), were included in the study. During the follow-up $35(16.82 \%)$ of 208 patients had died from pneumonia or complications due to pneumonia. According to multiple linear regression analysis, the following parameters were found to be predictors of mortality: Charlson comorbidity index value (odds ratio: $1.44,95 \%$ confidence interval: $1.132-1.1841, p=0.003$ ), chronic obstructive pulmonary disease (odds ratio: 0.292 , 95\% confidence interval: 0.094-1.149, $p=0.038$ ), congestive heart failure (odds ratio: 0.199, 95\% confidence interval: $0.051-0.782, p=0.021$ ), saturation value in arterial blood gas (odds ratio: $0.569,95 \%$ confidence interval: 0.804-0.939, $p<0.001$ ), intubation duration (odds ratio: $3.476,95 \%$ confidence interval: 1.880-6.425, $p<0.001$ ), hypertension (odds ratio: $3.449,95 \%$ confidence interval: $0.941-$ 12.649, $\mathrm{p}=0.042$ ), and the presence of diabetes mellitus (odds ratio: $3.116,95 \%$ confidence interval: 2.673-59.021, $p=0.046$ ).

Conclusion: Community-acquired pneumonia requiring intensive care unit is a clinical condition with high mortality in the elderly patient population. The presence of comorbid diseases and prolonged intubation time may be associated with higher mortality.

Key words: Pneumonia; Mortality; Geriatrics; Intensive Care Units 


\section{INTRODUCTION}

Community-acquired pneumonia (CAP) is a common disease among the elderly that has a high mortality rate. The estimated annual incidence of CAP in elderly patients is between 25 and 44 cases per 1000 people (1). The rate of hospitalization of elderly patients due to pneumonia is increasing, and CAP will be even more important in coming years, as $20 \%$ of the world population is expected to be elderly by 2050 (1). Compared to the population under 65 years, elderly patients over 65 have a higher incidence of CAP. This patient group almost always has some comorbid conditions, and mortality is higher than in the young population. The mortality rate in patients aged 65 years and over is between $10 \%$ and $30 \%$ (2). Many studies have been conducted on the relationship between pneumonia and short-term mortality in elderly patients. While in-hospital mortality was evaluated in some of these studies, others evaluated the 30-day mortality rate. Studies have shown that in-hospital mortality rates vary between $6 \%$ and $26 \%$ due to different inclusion and exclusion criteria (2-3).

Because of its atypical clinical presentation, varied epidemiology, and age-related changes in drug metabolism, CAP in the elderly is challenging for the clinician (1). Incorrect diagnosis of CAP may cause the patient to miss the best treatment opportunity and affect recovery and prognosis. Therefore, it is of great importance that we evaluate CAP progression in elderly patients in a timely and accurate manner.

Most studies investigating the factors affecting mortality in CAP have been conducted on under 65 years patients. Few studies have investigated these factors in elderly patients. The aim of this study was to determine the factors affecting mortality in elderly patients with CAP who were treated in an intensive care unit (ICU).

\section{MATERIALS AND METHODS}

The medical records of patients over the age of 65 who were hospitalized with a diagnosis of CAP between January 1, 2013, and February 29, 2020 at Baskent University, Faculty of Medicine, Adana Research Center, Turkey, were retrospectively reviewed. Patients with a clinical suspicion of respiratory tract infection (fever, cough, sputum, or dyspnea) and infiltration in the lung parenchyma detected in radiological imaging (direct radiography or computed tomography) were accepted as having a pneumonia diagnosis. Of the patients diagnosed with pneumonia, patients who were directly admitted to the ICU or hospitalized first and then admitted to ICU due to medical necessity were included in the study. The demographic data (age and gender) of the patients and their hemoglobin, white blood cell, thrombocyte, C-reactive protein, procalcitonin, arterial blood gas oxygen, carbon dioxide, saturation, and bicarbonate values were recorded. In addition, the pneumonia severity index, the Charlson comorbidity index (CCI) (4), the microbiology results (sputum, blood, and deep tracheal aspirate culture results), the CURB-65 score, the treatments they received, the length of stay in ICU, the duration of mechanical ventilation, the duration of hospitalization, and the proliferation of the infiltration on chest radiography were recorded. The records of patients who had died during their hospitalization and the patients who were discharged after completion of their treatment were compared, and the data related to mortality were determined. Additionally, by examining the records of patients who had died, the factors affecting mortality and the predictors of mortality were investigated.

Pneumonia occurring at least 48 hours after the patient was hospitalized or pneumonia developing within the first 48 hours after discharge were categorized as hospital-acquired pneumonia, and cases of pneumonia that emerged in the person's daily life other than for the reasons above were accepted as CAP (5). Acute respiratory failure was defined as the presence of severe respiratory distress accompanied by decreased oxygen saturation $(<92 \%)$ in room air, hypoxemia (partial oxygen pressure $<60$ 
$\mathrm{mm} \mathrm{Hg}$ ), or the need for invasive/noninvasive mechanical ventilation. Blood cultures were taken from all patients. Sputum culture was obtained from the patients who could give sputum samples, while deep tracheal aspirate culture was obtained from the intubated patients. Antibiotic treatment was initiated empirically for all patients in accordance with the guidelines and antibiotic treatment was rearranged, if necessary, according to the culture results (6-7).

\section{Study exclusion criteria}

Those who are under the age of 65, who develop pneumonia 48 hours after hospitalization, have a history of hospitalization within the last 48 hours when pneumonia is diagnosed, patients not treated in ICU during their hospitalization, bronchiectasis, chronic respiratory failure, renal failure requiring renal replacement therapy, use of immune suppressants, transplantation patients with end-stage chronic liver failure, those patients who did not have sufficient clinical information in their files, those who had another infection accompanying pneumonia during hospital admission, those who left the hospital voluntarily during the treatment, and patients who did not accept treatment, were excluded from the study.

\section{Statistical analyses}

Continuous variables are expressed as mean \pm standard deviation or median (range, interquartile range $[(Q R])$. Categorical data are presented as proportion. Chi-square test was used to analyze categorical parameters. Unpaired t-test was used to analyze continuous variables with normal distribution. Mann-Whitney $U$ test was used to analyze continuous variables with non-normal distribution. Independent determinants of mortality were determined by standard multiple linear regression analysis. Continuous variables were checked with Kolmogorov-Smirnov test to assess whether they were normally distributed. Values for $\mathrm{p}<0.05$ were assessed statistically significant. The statistical software, SPSS was used for statistical analysis (SPSS version 21.0 for Windows; SPSS, Inc., Chicago, IIlinois, US).

\section{RESULTS}

A total of 208 patients (78 [37.5\%] women) with a mean age of $75.11 \pm 5.59$ years were included in the study. The patients' baseline demographic, clinical and laboratory characteristics are summarized in Table-1. The median duration of ICU stay was 3 $(I Q R=2)$ days, and the median length of stay in the normal ward was $5.5(I Q R=4)$ days. Blood cultures were taken from all patients, and 48 (23.07\%) patients had a positive blood culture. Gram-positive cocci were found in the blood culture of 33 (68.75\%) patients, Gram-negative bacilli were observed in the blood culture of 10 (20.83\%) patients, and Acinetobacter growth was seen in the blood culture of $5(10.41 \%)$ patients. Sputum culture was obtained from 93 (44.71\%) patients; reproduction occurred in 72 (77.41\%) of these patients. The sputum culture of 51 (70.83\%) patients had Gram-positive cocci, 14 (19.44\%) had Gram-negative bacilli, and 7 (9.72\%) had Acinetobacter growth. Deep tracheal aspirate culture was obtained from $32 \mathrm{pa}$ tients. Acinetobacter reproduced in 11 (34.37\%) of these patients, while Gram-positive cocci growth in $9(28.12 \%)$ patients and Gram-negative bacillus growth in 6 (18.75\%) patients were observed. There was no growth in the deep tracheal aspirate culture of $6(18.75 \%)$ patients. A total of 32 patients (15.3\%) needed invasive mechanical ventilation. During the follow-up, 35 (16.82\%) of 208 patients died from pneumonia and pneumonia-related complications.

The comparison of the baseline clinical, demographic, and laboratory data of surviving and non-surviving patients summarized in Table-2. According to the multiple linear regression analysis, the $\mathrm{CCl}$ score, chronic obstructive pulmonary disease (COPD), congestive heart failure (CHF), ox- 
Table-1. Baseline clinical and laboratory parameters in the study population

\begin{tabular}{|c|c|}
\hline Age (year) & $75.11 \pm 6.69$ \\
\hline Female gender, n (\%) & $78(37.5)$ \\
\hline Charlson comorbidity index & $4.69 \pm 1.50$ \\
\hline CURB-65 score & $2.28 \pm 0.57$ \\
\hline Pneumonia severity index & $3.89 \pm 0.68$ \\
\hline Hypertension, n (\%) & $81(38.9)$ \\
\hline COPD, n (\%) & $59(28.4)$ \\
\hline Diabetes mellitus, n (\%) & $63(30.3)$ \\
\hline Asthma, n (\%) & $22(10.6)$ \\
\hline SVA, n (\%) & $37(17.8)$ \\
\hline Chronic liver disease, $\mathrm{n}(\%)$ & $4(1.9)$ \\
\hline Malignant, n (\%) & $12(5.8)$ \\
\hline Myasthenia gravis, $\mathrm{n}(\%)$ & $1(0.5)$ \\
\hline Dementia, n (\%) & $27(13)$ \\
\hline Heart failure, n (\%) & $29(13.9)$ \\
\hline Chronic kidney disease, n (\%) & $9(4.3)$ \\
\hline Hemoglobin (gr/dL) & $12.29 \pm 1.86$ \\
\hline White blood cell $\left(/ \mathrm{mm}^{3}\right)$ & $13243 \pm 7387$ \\
\hline Platelets $\left(100 / \mathrm{mm}^{3}\right)$ & $247(\mathrm{IQR}=158)$ \\
\hline Creatinine $(\mathrm{mg} / \mathrm{dL})$ & $1.19 \pm 0.52$ \\
\hline Sodium (mmol/L) & $137.89 \pm 5.81$ \\
\hline Potassium (mmol/L) & $4.24 \pm 0.52$ \\
\hline C-reactive protein (mg/L) & $103(\mathrm{IQR}=137)$ \\
\hline Procalcitonine (ng/mL) & $0.6 \mathrm{IOR}=1.3)$ \\
\hline Arterial blood gas $\mathrm{pH}$ & $7.42 \pm 0.06$ \\
\hline Arterial blood gas oxygen saturation (\%) & $88.08 \pm 7.70$ \\
\hline Arterial blood gas oxygen $(\mathrm{mm} \mathrm{Hg})$ & $58.60 \pm 11.30$ \\
\hline Arterial blood gas carbondioxide (mm Hg) & $33.95 \pm 9.20$ \\
\hline Arterial blood gas bicarbonate $(\mathrm{mmol} / \mathrm{L})$ & $23.00 \pm 4.01$ \\
\hline
\end{tabular}

COPD: Chronic obstructive pulmonary disease, IQR: Inter quartile range, SVA: Serebro vascular accident ygen saturation value in arterial blood gas at first admission, intubation time, hypertension, and diabetes mellitus were predictors of mortality. The regression analysis results are summarized in Table-3.

\section{DISCUSSION}

According to the results of our study, the mortality from CAP in patients over 65 years old was $16.82 \%$. The oxygen saturation value in arterial blood gas at first admission, COPD, CHF, hypertension, diabetes mellitus, intubation time, and the $\mathrm{CCl}$ score were predictors of mortality.

In elderly patients, both in- and out-of-hospital CAP mortality is higher than in non-elderly patients. In a study by Mangen et al., in which 562 patients with CAP over the age of 65 were examined, the annual mortality after discharge in elderly patients was found to be six times higher than in non-elderly patients. Their study also observed a 16\% higher reduction in the quality of life of elderly patients after discharge (8). The increase in mortality in elderly patients may be due to comorbid conditions. It is well known that elderly patients with high comorbidity have low resistance to infections. In various studies, chronic respiratory disease, COPD, diabetes mellitus, chronic cardiovascular diseases, and chronic neurological diseases have been found to be the most common comorbid diseases observed in elderly patients during their pneumonia treatment (9-10). In addition, these comorbid conditions have been associated with prolonged hospitalization, prolonged ICU admission, sepsis, readmission, and mortality (10-11). The results of our study are consistent with the literature, and these comorbid conditions were observed with a higher incidence in the group that died in our study. Moreover, COPD, CHF, hypertension, and diabetes mellitus were factors that directly affected mortality in our study (9-11).

In elderly patients, the mortality of conditions requiring respiratory ICU admission may be high. In a recent study, the most common respiratory rea- 
Table-2. Comparison of baseline clinical, demographic and laboratory values between groups with and without exitus

\begin{tabular}{|c|c|c|c|}
\hline & No exitus ( $n=173$ ) & Exitus $(n=35)$ & $p$ \\
\hline Age (year) & $75.27 \pm 6.91$ & $74.31 \pm 5.54$ & 0.445 \\
\hline Female gender, n (\%) & $69(39.9)$ & $9(25.7)$ & 0.114 \\
\hline Charlson comorbidity index & $4.55 \pm 1.46$ & $5.40 \pm 1.51$ & 0.002 \\
\hline Pneumonia severity index & $3.76 \pm 0.61$ & $4.57 \pm 0.55$ & $<0.001$ \\
\hline CURB-65 score & $2.08 \pm 0.26$ & $3.29 \pm 0.62$ & $<0.001$ \\
\hline Hypertension, n (\%) & $63(36.4)$ & $18(51.4)$ & 0.097 \\
\hline COPD, n (\%) & $43(24.9)$ & $16(45.7)$ & 0.013 \\
\hline Diabetes mellitus, n (\%) & $50(28.9)$ & $13(37.1)$ & 0.333 \\
\hline Asthma, n (\%) & $18(10.4)$ & $4(11.4)$ & 0.77 \\
\hline SVA, n (\%) & $25(14.5)$ & $12(34.3)$ & 0.005 \\
\hline Chronic liver disease, n (\%) & $4(2.3)$ & $0(0)$ & NA \\
\hline Malignant, n (\%) & $11(6.4)$ & $1(2.9)$ & 0.695 \\
\hline Myasthenia gravis, n (\%) & $0(0)$ & $1(2.9)$ & NA \\
\hline Dementia, n (\%) & $18(10.4)$ & $9(25.7)$ & 0.014 \\
\hline Heart failure, n (\%) & 18 (10.4) & 11(31.4) & 0.001 \\
\hline Chronic kidney disease, n (\%) & $8(4.6)$ & $1(2.9)$ & 0.534 \\
\hline Hemoglobin (gr/dL) & $12.33 \pm 1.89$ & $12.06 \pm 1.73$ & 0.442 \\
\hline White blood cell $\left(/ \mathrm{mm}^{3}\right)$ & $13077 \pm 7543$ & $14062 \pm 6610$ & 0.473 \\
\hline Platelets $\left(100 / \mathrm{mm}^{3}\right)$ & $238(\mathrm{IQR}=158)$ & $284(I Q R=149)$ & 0.172 \\
\hline Creatinine (mg/dL) & $1.06 \pm 0.46$ & $1.27 \pm 0.96$ & 0.054 \\
\hline Sodium (mmol/L) & $137.56 \pm 5.05$ & $139.51 \pm 8.53$ & 0.07 \\
\hline Potassium (mmol/L) & $4.23 \pm 0.48$ & $4.3 \pm 0.68$ & 0.473 \\
\hline C-reactive protein $(\mathrm{mg} / \mathrm{L})$ & $96(I Q R=132)$ & $154(\mid \mathrm{QR}=118)$ & 0.007 \\
\hline Procalcitonine (mg/L) & $0.46(I Q R=1.1)$ & $1.3(\mathrm{IQR}=1.4)$ & $<0.001$ \\
\hline Arterial blood gas $\mathrm{pH}$ & $7.42 \pm 0.05$ & $7.38 \pm 0.09$ & $<0.001$ \\
\hline Arterial blood gas oxygen saturation (\%) & $89.79 \pm 5.8$ & $79.68 \pm 10.18$ & $<0.001$ \\
\hline Arterial blood gas oxygen $(\mathrm{mm} \mathrm{Hg})$ & $60.62 \pm 10.54$ & $48.65 \pm 9.6$ & $<0.001$ \\
\hline Arterial blood gas carbondioxide $(\mathrm{mm} \mathrm{Hg})$ & $33.17 \pm 6.88$ & $37.77 \pm 16.05$ & 0.007 \\
\hline Arterial blood gas bicarbonate $(\mathrm{mmol} / \mathrm{L})$ & $22.97 \pm 2.95$ & $23.15 \pm 7.33$ & 0.807 \\
\hline
\end{tabular}

COPD: Chronic obstructive pulmonary disease, IQR: Interquartile range, NA: Non-available, SVA: Serebro vascular accident 
Table-3. Multiple linear regression analysis of mortality with clinical variables.

\begin{tabular}{|l|c|c|c|c|c|}
\hline & Beta coefficient & OR & $p$ & \multicolumn{2}{|c|}{ 95\% Confidence Interval } \\
\hline & & & & \multicolumn{2}{|c|}{ Lower } \\
\hline Charlson comorbidity index & 0.367 & 1.444 & 0.003 & 1.132 & 1.841 \\
\hline COPD & -1.231 & 0.292 & 0.038 & 0.094 & 1.149 \\
\hline CHF & -1.615 & 0.199 & 0.021 & 0.051 & 0.782 \\
\hline Saturation in arterial blood gas* & -0.141 & 0.869 & $<0.001$ & 0.804 & 0.939 \\
\hline Intubation duration time & 1.246 & 3.476 & $<0.001$ & 1.880 & 6.425 \\
\hline Hypertension & 1.238 & 3.449 & 0.042 & 0.941 & 12.649 \\
\hline Diabetes mellitus & 1.137 & 3.116 & 0.046 & 0.817 & 11.885 \\
\hline Dementia & 2.531 & 12.560 & 0.001 & 2.673 & 59.021 \\
\hline
\end{tabular}

*First application blood gas, CHF: Congestive heart failure, COPD: Chronic obstructive pulmonary disease, OR: Odds ratio

son for admission to ICU in patients over 65 years of age was pneumonia, with a $65 \%$ incidence. In the same study, albumin levels, APACHE II scores, and vasopressor requirements were the most important determinants of mortality (12). Some studies have investigated the predictors of mortality in CAP in elderly patients. In a retrospective study by Öcal et al. that included 186 patients (mean age $63.7 \pm 20.5$ years; 114 patients $>65$ years old), the determinants of mortality in geriatric patients hospitalized with a diagnosis of pneumonia were investigated. According to their results, age, prolonged ICU admission, anemia, hypoglycemia, hypoalbuminemia, and increased lactate dehydrogenase levels were risk factors for mortality. Furthermore, the mean length of ICU stay was longer for the geriatric patients (13).

According to another result of our study, the $\mathrm{CCl}$ score may be a predictor of mortality. Some studies have investigated mortality predictors in patients with CAP. Among these studies, the most frequently investigated were the pneumonia severity index and the CURB-65 score. Although there are conflicting results in different studies, the accuracy rate of these risk scoring systems decreases in elderly patients $(1,14)$. In a study by Franzen et al. that included 108 patients with CAP, the $\mathrm{CCl}$ score was not associated with undesirable, poor results. The reason why the results of this study differ from our study may be that it included patients from all age groups, and none of the patients required ICU hospitalization. Also, the pneumonia severity index was found to be a predictor of undesirable outcomes (15). In another study by Nguyen et al. (median age 79) with 565 patients with $\mathrm{CAP}$, the $\mathrm{CCl}$ score was associated with high in-hospital mortality (Odds ratio [OR]:1.28; 95\% Confidence interval [CI]: 1.07-1.53) (16). The fact that this study investigated an elderly patient group may account for the similar results to our study.

The strength of the study is that it is a clinical study and gives direct information about the factors affecting mortality in elderly patients on the other hand the weakness of our study is that it was a single center study.

Limitations of the study: The biggest limitation of our study is that it was conducted with a small number of patients. These results should be confirmed with multi-center studies including more patients. The retrospective study design is another limitation. The difference between the time of admission to the hospital after the onset of symptoms and the different treatment initiation times of the patients included in the study may also have affected the results. 
Conclusion: According to the results of our study, the mortality of CAP in patients over 65 years old was $16.8 \%$. The predictors of mortality may be the values of some parameters during initial admission to the hospital, such as blood gas saturation and $\mathrm{CCl}$ score, the intubation period and the presence of COPD, CHF, hypertension, and diabetes mellitus.

Conflict of Interest, Disclosure Statement: The authors declare that they have no conflicts of interest. The authors have indicated the have no finan-

\section{REFERENCES}

1. Cillóniz $C$, Dominedò $C$, Pericàs $J M$, Rodriguez-Hurtado D, Torres A. Community-acquired pneumonia in critically ill very old patients: a growing problem. Eur Respir Rev. 2020;29(155). (PMID: 32075858).

2. Arnold FW, Reyes Vega AM, Salunkhe V, et al. Older Adults Hospitalized for Pneumonia in the United States: Incidence, Epidemiology, and Outcomes. J Am Geriatr Soc. 2020;68(5):1007-1014. (PMID: 31916246).

3. Pessoa E, Bárbara C, Viegas L, Costa A, Rosa M, Nogueira P. Factors associated with in-hospital mortality from community-acquired pneumonia in Portugal: 2000-2014. BMC Pulm Med. 2020;20(1):18. (PMID: 31964385).

4. Charlson M, Szatrowski TP, Peterson J, Gold J. Validation of a combined comorbidity index. J Clin Epidemiol. 1994;47(11):1245-1251. (PMID: 7722560).

5. Lanks CW, Musani Al, Hsia DW. Community-acquired Pneumonia and Hospital-acquired Pneumonia. Med Clin North Am. 2019;103(3):487-501. (PMID: 30955516).

6. Musher DM, Thorner AR. Community-acquired Pneumonia. N Engl J Med. 2014;371(17):1619-1628. (PMID: 25337751).

7. Metlay JP, Waterer GW, Long AC, et al. Diagnosis and Treatment of Adults with Community-acquired cial relationships relevant to this article to disclose

Ethical Approval: All procedures performed in this study involving human participants were in accordance with the ethical standards of the institutional and/or national research committee and with the 1964 Helsinki declaration and its later amendments or comparable ethical standards. This study was approved by Baskent University Institutional Review Board (Project no: KA20/39) and supported by Baskent University Research Fund.

Acknowledgments: None

Pneumonia. An Official Clinical Practice Guideline of the American Thoracic Society and Infectious Diseases Society of America. Am J Respir Crit Care Med. 2019;200(7):e45-e67. (PMID: 31573350).

8. Mangen MJ, Huijts SM, Bonten MJ, de Wit GA. The impact of community-acquired pneumonia on the health-related quality-of-life in elderly. BMC Infect Dis. 2017;17(1):208. (PMID: 28292280).

9. Laporte L, Hermetet C, Jouan Y, et al. Ten-year trends in intensive care admissions for respiratory infections in the elderly. Ann Intensive Care. 2018;8(1):84. (PMID: 30112650).

10. Luna CM, Palma I, Niederman MS, et al. The impact of age and comorbidities on the mortality of patients of different age groups admitted with community-acquired pneumonia. Ann Am Thorac Soc. 2016;13(9):1519-1526. (PMID: 27398827).

11. Cillóniz C, Polverino E, Ewig $S$, et al. Impact of age and comorbidity on cause and outcome in community-acquired pneumonia. Chest. 2013;144(3):9991007. (PMID: 23670047).

12. Yazıcı O, Ceylan E, Polatıı M, Gülen ŞT, Karadağ F. Clinical Characteristics and Factors Affecting Mortality in Elderly Patients Admitted to Respiratory Intensive Care Unit. Turkish Journal of Geriatrics. 2020;23(1):27-34. (DOI: 10.31086/tjgeri.2020.134) 
13. Öcal N, Doğan D, Taşkın G, Yıldız B, Özer S, Yamanel L. Continual Assessment of Mortality Risk Factors in Geriatric Patients Hospitalized in Intensive Care Due to Pneumonia. Turkish Journal of Geriatrics. 2016;19(1):1-8

14. Chen J-H, Chang S-S, Liu JJ, et al. Comparison of clinical characteristics and performance of pneumonia severity score and CURB-65 among younger adults, elderly and very old subjects. Thorax. 2010;65(11):971-977. (PMID: 20965934).
15. Franzen D, Lim M, Bratton DJ, Kuster SP, Kohler M. The Roles of the Charlson Comorbidity Index and Time to First Antibiotic Dose as Predictors of Outcome in Pneumococcal Community-Acquired Pneumonia. Lung. 2016;194(5):769-775. (PMID: 27405854).

16. Nguyen MTN, Saito N, Wagatsuma Y. The effect of comorbidities for the prognosis of community-acquired pneumonia: an epidemiologic study using a hospital surveillance in Japan. BMC Res Notes. 2019;12(1):817. (PMID: 31856910). 